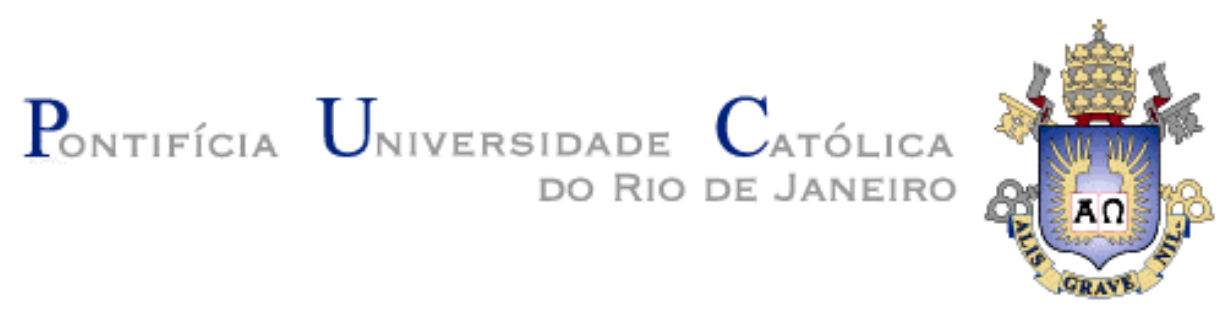

Fábio de Oliveira Paula

Fatores que Influenciam o Desempenho das Firmas Brasileiras: um modelo integrativo

Dissertação de Mestrado

Dissertação apresentada como requisito parcial para obtenção do título de Mestre pelo Programa de Pós-Graduação em Administração da PUC-Rio.

Orientador: Prof. Jorge Ferreira da Silva

Rio de Janeiro, agosto de 2006 


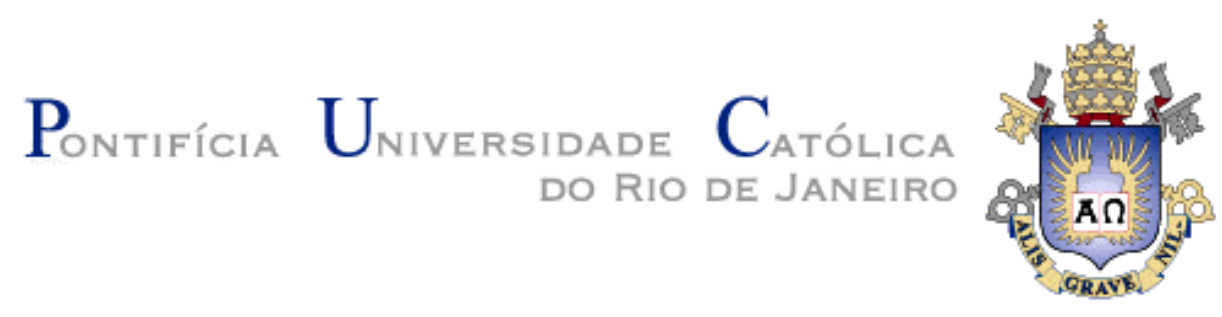

Fábio de Oliveira Paula

\section{Fatores que Influenciam o Desempenho das Firmas Brasileiras: um modelo integrativo}

Dissertação apresentada como requisito parcial para obtenção do título de Mestre pelo Programa de PósGraduação em Administração da PUC-Rio. Aprovada pela Comissão Examinadora abaixo assinada.
Prof. Jorge Ferreira da Silva Orientador PUC-Rio

Prof. Marie Agnes Chauvel PUC-Rio

Prof. Jorge Ferreira da Silva

PUC-Rio

Prof. Angela Maria Cavalcanti da Rocha UFRJ

Prof. João Pontes Nogueira Coordenador(a) Setorial do Centro de Ciências Sociais - PUC-Rio 
Todos os direitos reservados. É proibida a reprodução total ou parcial do trabalho sem autorização da universidade, do autor e do orientador.

\section{Fábio de Oliveira Paula}

Brasileiro, solteiro, 26 anos. Formado em Engenharia de Computação pela PUC-Rio, fez intercâmbio por um ano na University of Wisconsin - Madison nos EUA. Trabalhou com informática por aproximadamente 4 anos e hoje trabalha numa empresa de varejo na parte de processos de mercado. Tem interesse nas áreas de marketing, processos e planejamento estratégico.

Ficha Catalográfica

Paula, Fábio de Oliveira

Fatores que Influenciam o Desempenho das Firmas Brasileiras: um modelo integrativo / Fábio de Oliveira Paula ; orientador: Jorge Ferreira da Silva. - Rio de Janeiro : PUC-Rio, Departamento de Administração, 2006.

119 f. : il. ; $30 \mathrm{~cm}$

Dissertação (mestrado) - Pontifícia Universidade Católica do Rio de Janeiro, Departamento de Administração.

Inclui referências bibliográficas.

1. Administração - Teses. 2. Estratégia. 3. Desempenho. 4. Modelo integrativo. 5. Modelagem quantitativa. 6. Maximum likelihood estimation. I. Silva, Jorge Ferreira da. II. Pontifícia Universidade Católica do Rio de Janeiro. Departamento de Administração. III. Títıln 


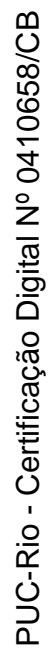

Para meus pais, Roberto e Neide, por tudo o que fizeram por mim; e para meus tios, Jarbas e Jacyra in memorian. 


\section{Agradecimentos}

Ao meu orientador, Jorge Ferreira da Silva, pelo estímulo, por todo conhecimento que me passou e pela confiança de que eu podia realizar um bom trabalho.

À PUC-Rio, por todos os recursos que me disponibilizaram para a realização deste trabalho que, sem os quais, não poderia ser realizado.

A todos os professores que me deram aula no mestrado e que, de alguma forma, contribuíram para a minha formação.

Aos meus pais, Roberto e Neide, pelo carinho, pela educação e por considerarem que o meu sucesso também é o sucesso deles.

Aos meus colegas de turma que compartilharam comigo todo o esforço e as alegrias de se fazer o mestrado.

A todos os amigos e familiares que acreditaram em mim e que sempre estiveram do meu lado. 


\section{Resumo}

Paula, Fábio de Oliveira; Silva, Jorge Ferreira da. Fatores que Influenciam o Desempenho das Firmas Brasileiras: um modelo integrativo. Rio de Janeiro, 2006. 119p. Dissertação de Mestrado Departamento de Administração, Pontifícia Universidade Católica do Rio de Janeiro.

O objetivo deste trabalho é responder a seguinte pergunta: Quais são os fatores que influenciam o desempenho das firmas? Para isso, foi desenvolvido um modelo integrativo utilizando o conhecimento produzido pelas escolas consagradas posicionamento e resource-based. Esse modelo prega que o desempenho é influenciado pelos recursos da firma, pela estratégia que ela adota e pela estrutura da indústria onde ela está inserida. Por sua vez, esses três fatores são influenciados pelo ambiente que cerca a firma. Esse modelo, então, foi testado pelo método de modelagem de equações estruturais Maximum likelihood estimation (MLE) com dados simulados. Juntamente com o modelo proposto, foram testados dois modelos consagrados na literatura. Um baseado nas teorias da escola posicionamento (adaptado de Hill \& Deeds, 1996), e o outro baseado nas teorias da escola resource-based (adaptado de Schroeder et al., 2002). O teste do modelo proposto mostrou que o desempenho realmente é afetado pelos três fatores citados acima, porém os indicadores de fit mostraram que o modelo não representa bem os dados utilizados para testá-lo. Em contrapartida, os dois modelos consagrados na literatura tiveram indicadores de fit bem melhores, o que mostra que eles representam melhor os dados simulados usados para fazer o teste com a técnica estatística utilizada.

\section{Palavras-chave}

Estratégia; desempenho; modelo integrativo; modelagem quantitativa; maximum likelihood estimation. 


\section{Abstract}

Paula, Fábio de Oliveira; Silva, Jorge Ferreira da (Advisor). Factors that Influence the Performance of the Brazilian Firms: an integrative model. Rio de Janeiro, 2006. 119p. Dissertation - Departamento de Administração, Pontifícia Universidade Católica do Rio de Janeiro.

This study will address the proposed research question: Which factors influence the performance of the firms? To answer that question, an integrative model was developed based on the knowledge produced by the consecrated positioning school and resource-based view. This model says that the firm resources, the strategy adopted by the firm and the industry structure are the factors that influence the performance. In addition, the tree factors cited above are influenced by the environment. This model was, then, tested by the structural equation modeling method Maximum likelihood estimation (MLE) with simulated data. Altogether with the proposed model, two consecrated by literature models were tested. The first one is based on positioning school theories (adopted from Hill \& Deeds, 1996) and the second one is based on resource-based view theories (adopted from Schroeder et al., 2002). The poposed model test demonstrated that, although performance is really affected by the tree factors cited above, the fit indicators showed that the model doesn't represent very well the data used to test it. On the other hand, the two consecrated by literature models had much better fit indicators. It shows that they represent much better the simulated data used to test them with the statistical method used.

\section{Keywords}

Strategy; performance; integrative model; quantitative modeling; maximum likelihood estimation. 


\section{Sumário}

1 Introdução 13

1.1. Contextualização da pesquisa 13

1.2. Problema de pesquisa 14

$\begin{array}{ll}\text { 1.3. Objetivos } & 15\end{array}$

1.4. Delimitação do estudo 17

$\begin{array}{ll}\text { 1.5. Relevância do estudo } & 17\end{array}$

2 Referencial teórico 19

2.1. Revisão da Literatura 19

2.1.1. O que é Estratégia? 19

2.1.1.1. Estratégia são 5 P's 22

2.1.1.2. Estratégia competitiva e fatores importantes para formulação 24

2.1.2. O que é Desempenho? 25

2.1.2.1. Medidas contábeis 26

2.1.2.2. Um modelo de desempenho em camadas 28

2.1.2.3. Uma variação do modelo em camadas: a interação entre os desempenhos 29

2.1.3. Por dentro da estratégia: resolvendo o modelo SWOT 31

2.1.3.1. Escola posicionamento 32

2.1.3.2. A visão "Resource-based" (RBV) 46

2.1.3.3. Integração entre RBV e a escola posicionamento 55

2.1.3.4. Fatores ambientais 56

2.1.3.5. Modelo proposto e hipóteses de pesquisa 57

3 Metodologia 66

3.1. Linha epistemológica 66

3.2. Metodologia no campo da estratégia 67

$\begin{array}{ll}\text { 3.3. Metodologia para análise dos dados } & 74\end{array}$ 
3.3.1. Análise de Fator 75

3.3.1.1. Interpretação dos resultados $\quad 77$

3.3.2. Modelagem de Equações Estruturais (MEE) 78

3.4. Caracterização da amostra 81

4 Análise e Interpretação dos Dados 84

4.1. Fase 1 - Redução das variáveis do modelo proposto 85

4.1.1. Construto Fatores Ambientais 85

4.1.2. Construto Aprendizado Interno 87

4.1.3. Construto Aprendizado Externo 88

4.1.4. Construto Estratégia 89

4.1.5. Construto Estrutura da Indústria 90

4.1.6. Construto Processos e Equipamentos Proprietários 91

4.1.7. Construto Desempenho 92

4.2. Fase 2 - Rodando o modelo 1 com MLE 93

4.3. Fase 3 - Rodando o modelo 2 com MLE 98

4.4. Fase 4 - Rodando o modelo 3 com MLE 101

4.5. Fase 5 - Comparando os resultados dos três modelos 104

5 Considerações Finais 106

5.1. Conclusões 106

$\begin{array}{ll}\text { 5.2. Limitações da pesquisa } & 108\end{array}$

6 Referências bibliográficas $\quad 111$

7 Anexo 116 


\section{Lista de figuras}

Figura 1 De estratégia pretendida a realizada - adaptada de Mintzberg et al. (2000)

Figura 2 Contexto da formulação da estratégia competitiva (Porter, 1980)

Figura 3 As camadas das medidas de desempenho organizacional (Venkatraman \& Ramanujam ,1986)

Figura 4 A iteração dos construtos de desempenho 31

Figura 5 Perspectiva de Porter (Hill \& Deeds, 1996) 32

Figura 6 llustração de um ciclo de vida de produto (Walker et al.,1996) 36

Figura 7 Revitalizando mercados em declínio(Aaker, 1984)

Figura 8 Modelo de explicação do desempenho de acordo com o posicionamento e a estrutura da indústria

Figura 9 Ciclo de vida da Capacitação (Helfat e Winter,2003)

Figura 10 Modelo de explicação de desempenho baseado no aprendizado da firma e nos recursos (adaptado de Schroeder et al, 2002)

Figura 11 Modelo de Spanos \& Lioukas (2001). Integrando ambas as escolas para tentar explicar o desempenho das firmas.

Figura 12 Fatores ambientais (Austin, 1990)

Figura 13 Modelo proposto

Figura 14 Modelo simplificado

Figura 15 Diagrama de caminho (Arbuckle at al., 1999 - pp. 125)

Figura 16 Resultado do Modelo 2

Figura 17 Resultado do Modelo 3 


\section{Lista de quadros}

Quadro 1 A atuação de cada força em cada tipo de indústria

Quadro 2 Tipologia de classificação de estudos em estratégia

(Venkatraman \& Camillus, 1984)

69

Quadro 3 Critérios para escolha de método de operacionalização do construto estratégia (Hambrick, 1980)

Quadro 4 Um resumo dos componentes chave da validação de construto (Venketraman e Grant, 1986)

Quadro 5 Métodos utilizados na literatura para se fazer validação de estudos em estratégia (Scandura e Williams, 2000)

Quadro 6 Significâncias das relações entre os construtos e variáveis

- modelo 1

Quadro 7 Correlações entre construtos e entre os construtos e variáveis - modelo 1

Quadro 8 Significâncias das relações entre os construtos e variáveis

- modelo 2

Quadro 9 Correlações entre construtos e entre os construtos e variáveis - modelo 2

Quadro 10 Significâncias das relações entre os construtos e variáveis

- modelo 3

Quadro 11 Correlações entre construtos e entre os construtos e variáveis - modelo 3

Quadro 12 Comparação entre os modelos com relação ao fit

Quadro 13 Matriz de fatores rodados do construto Fatores Ambientais 116

Quadro 14 Matriz de fatores rodados do construto Aprendizado

Interno

Quadro 15 Matriz de fatores rodados do construto Aprendizado

Externo

Quadro 16 Matriz de fatores rodados do construto Estratégia

Quadro 17 Matriz de fatores rodados do construto Estrutura da Indústria 
Quadro 18 Matriz de fatores rodados do construto Processos e Equipamentos Proprietários 118

Quadro 19 Matriz de fatores rodados do construto Desempenho 119 